\title{
Antimicrobial resistance of Neisseria gonorrhoeae in Germany: low levels of cephalosporin resistance, but high azithromycin resistance
}

Susanne Buder ${ }^{1 *+} \mathbb{D}$, Sandra Dudareva ${ }^{2,3^{*}+}$, Klaus Jansen$^{2}$, Anna Loenenbach², Sergejs Nikisins ${ }^{2,4,5}$, Andrea Sailer ${ }^{2}$, Eva Guhl', Peter K. Kohl', Viviane Bremer ${ }^{2}$ and GORENET study group

\begin{abstract}
Background: The widespread antimicrobial resistance of Neisseria gonorrhoeae is a serious problem for the treatment and control of gonorrhoea. Many of the previously effective therapeutic agents are no longer viable. Because N. gonorrhoeae infections are not reportable in Germany, only limited data on disease epidemiology and antimicrobial susceptibility patterns are available. The Gonococcal Resistance Network (GORENET) is a surveillance project to monitor trends in the antimicrobial susceptibility of N. gonorrhoeae in Germany in order to guide treatment algorithms and target future prevention strategies.
\end{abstract}

Methods: Between April 2014 and December 2015, data on patient-related information were collected from laboratories nationwide, and susceptibility testing was performed on $537 \mathrm{~N}$. gonorrhoeae isolates forwarded from the network laboratories to the Conciliar Laboratory for gonococci. Susceptibility results for cefixime, ceftriaxone, azithromycin, ciprofloxacin and penicillin were defined according to EUCAST 4.0 standards. Percentages, medians and interquartile ranges (IQR) were calculated.

Results: Altogether, $90 \%$ of isolates were from men. The median age was 32 (IQR 25-44) years for men and 25 (IQR 22-40) years for women ( $p$-value $<0.001)$. The most frequently tested materials among men were urethral (96.1\%) and rectal swabs (1.7\%), and among women, it was mainly endocervical and vaginal swabs (84.3\%). None of the isolates were resistant to ceftriaxone. Furthermore, 1.9\% (in 2014) and 1.4\% (in 2015) of the isolates were resistant to cefixime, $11.9 \%$ and $9.8 \%$ showed resistance against azithromycin, $72.0 \%$ and $58.3 \%$ were resistant to ciprofloxacin, and $29.1 \%$ and $18.8 \%$ were resistant to penicillin.

Conclusions: Resistance to ceftriaxone was not detected, and the percentage of isolates with resistance to cefixime was low, whereas azithromycin resistance showed high levels during the observation period. The rates of ciprofloxacin resistance and penicillin resistance were very high across Germany. Continued surveillance of antimicrobial drug susceptibilities for $\mathrm{N}$. gonorrhoeae remains highly important to ensure efficient disease management.

Keywords: Neisseria gonorrhoeae, Gonorrhoea, Antimicrobial resistance, Resistance surveillance

\footnotetext{
*Correspondence: dr.susanne.buder@web.de; DudarevaS@rki.de

${ }^{\dagger}$ Equal contributors

'German Consiliary Laboratory for Gonococci, Department of Dermatology

and Venerology, Vivantes Hospital Berlin, Berlin, Germany

${ }^{2}$ Department for Infectious Disease Epidemiology, Robert Koch Institute,

Berlin, Germany

Full list of author information is available at the end of the article
} 


\section{Background}

The worldwide development of antimicrobial resistance in Neisseria gonorrhoeae is a serious problem for the treatment and control of gonorrhoea. Treatment opportunities are dramatically limited because many of the previously recommended therapeutic agents are no longer effective.

The World Health Organization (WHO) [1], the Centers for Disease Control and Prevention (CDC) [2], and the European Centre for Disease Prevention and Control (ECDC) [3] have called for action to confine the spread of multidrug-resistant $N$. gonorrhoeae by enhancing the surveillance system of $N$. gonorrhoeae susceptibility testing and by strengthening laboratory capacities to perform culture and antimicrobial resistance testing. Representative coverage of collected data, unified $N$. gonorrhoeae susceptibility testing methods and interpretation standards are of great importance [4-6].

Extended spectrum cephalosporins (ESCs) are the last agents effective against $N$. gonorrhoeae. However, resistance to ESCs is increasingly common [7-13], causing concern that the efficacy of this substance group could expire in the near future [5-10]. Due to the worldwide rising resistance of $N$. gonorrhoeae against cefixime in recent years, the treatment guidelines needed to be changed accordingly. Thus, cefixime is no longer recommended as first-line therapy in many countries [14-16] and new therapy guidelines and action plans have been developed to keep gonorrhoea a treatable disease. In 2014 the German STI Society implemented new therapy guidelines for Germany. These guidelines recommend the use of the ESC ceftriaxone (1000 mg single dose i.m. or i.v.), now in combination with azithromycin as dual therapy (1.5 g single dose p.o.), as the first-line treatment [17]. The use of cefixime ( $800 \mathrm{mg}$ single dose p.o.) should be reserved for cases where parenteral treatment is not possible and ideally after susceptibility testing.

Because gonorrhoea is not a notifiable disease in Germany, only very limited data about epidemiology and antimicrobial resistance against $N$. gonorrhoeae are available. Furthermore, no standard operating procedures or unified protocols for N. gonorrhoeae susceptibility testing have been established in Germany. To date, information about $N$. gonorrhoeae susceptibility can be derived from several cross-sectional, regionally limited studies [18-21] through the German antibiotic resistance surveillance programme (ARS) and 100-120 isolates submitted to Euro-GASP yearly through the Consiliary Laboratory (CL) for gonococci [22]. Within this Euro-GASP collection, isolates resistant against ceftriaxone (MIC $>0.125 \mathrm{mg} / \mathrm{L}$ ) have been observed in Germany. Resistance against ceftriaxone was shown by $6.5 \%(n=7)$ of all German Euro-GASP isolates in 2011, while in the following years 2012-2014, one isolate per year (1\%) could be identified with ceftriaxone resistance. Cefixime resistance (MIC $>0.125 \mathrm{mg} / \mathrm{L}$ ) in German EuroGASP isolates ranged between $5.7 \%$ and $12.9 \%(n=13$, $11.9 \%$ in $2010 ; n=11,10.2 \%$ in $2011 ; n=6,5.7 \%$ in 2012 ; $n=13,12.9 \%$ in 2013) [22]. In 2014, there was no detection of cefixime-resistant isolates in Euro-GASP.

To implement continuous routine data collection on epidemiology and antimicrobial susceptibility testing for N. gonorrhoeae in German laboratories (aim 1) and to collect isolates for testing in the German CL for gonococci with unified methodology (aim 2), we set up a $N$. gonorrhoeae resistance network (GORENET).

We analysed the data and isolates collected through GORENET in 2014 and 2015 to guide treatment algorithms and targeted prevention strategies in Germany.

\section{Methods}

To characterise laboratories testing for $N$. gonorrhoeae in Germany and indicate laboratories for recruitment, we performed a cross-sectional survey between June and August 2013, as described previously [23]. From the laboratories that expressed an interest in participating, we recruited private and hospital laboratories for GORENET, prioritizing those with a wider catchment area and a higher number of $N$. gonorrhoeae tests per quarter. The laboratories in Germany have no predefined catchment areas, and practitioners are free to choose laboratories for cooperation. For a better geographical coverage of the data, we strove to recruit laboratories from all regions in Germany and laboratories that use any gradient Etest for $N$. gonorrhoeae susceptibility testing, at least for azithromycin, ceftriaxone and cefixime. Participation was voluntary, and there was no financial compensation for laboratories to participate in the study. The data collection protocol was confirmed by the data protection officer at the Robert Koch Institute (RKI), Berlin. Additional approval from an ethics committee was deemed to be unnecessary.

\section{Continuous routine data collection}

From GORENET network laboratories, continuous routine data on all samples tested for $N$. gonorrhoeae antimicrobial susceptibility were collected between April 2014 and December 2015. The network laboratories submitted data to the RKI (further labelled as samples). The collected data included sample identification number, information on test results, sampled material (urethral swab, urine, vaginal swab, cervical swab, rectal swab, pharyngeal swab, and other material), date of sampling, date of testing, district code, gender and year of birth. If the district code of the patient was not available, we used the code of the laboratory instead. Based on the year of birth, we calculated the person's age at the time of sampling. If the date of testing was 
not available, we used the date when the isolate was received in the CL.

Data were transmitted electronically to the RKI. Laboratories entered the data either in an online questionnaire (VOXCO Command Center 3) or in a preformatted Excel spreadsheet (.xls). Data on N. gonorrhoeae susceptibility from laboratories willing to participate in GORENET but already submitting their susceptibility data (including data on a wide range of other agents) to the German Antibiotic Resistance Surveillance Programme (ARS) were extracted from the ARS database. We performed plausibility checks on all reported data.

Based on the district codes, we described the geographical distribution of the samples tested for $N$. gonorrhoeae antimicrobial susceptibility. Each district code or respective 3-digit postal code corresponded to one district in Germany. We used samples tested for susceptibility from participating laboratories to describe tested persons by gender, age, sampled material and treating specialist. Susceptibility results from participating laboratories are used for national surveillance, but not presented in this paper.

An overview of the analysis of samples tested for $N$. gonorrhoeae susceptibility in network laboratories is given in Table 1.

\section{Isolate collection and susceptibility testing}

The network laboratories were asked to send $N$. gonorrhoeae isolates from the samples tested for antimicrobial susceptibility between April 2014 and December 2015 to the CL for extended and comparative susceptibility testing (further labelled as isolates). There were no criteria used to preselect isolates that should be sent to CL. The sample identification number was used to link isolates to samples. For all received isolates, we confirmed $N$. gonorrhoeae by using a combination of culture on non-selective agar medium, rapid oxidase production assays and determining the presence of Gram-negative diplococci using microscopy and the Phadebact Monoclonal GC OMNI Test (Pharmacia Diagnostics, Piscataway, NJ, USA). Susceptibility testing was performed and
MIC were detected by using Etest (bioMérieux SA, Marcy-l'Étoile, France) according to the manufacturer's instructions for ceftriaxone, cefixime, azithromycin, penicillin and ciprofloxacin. To define resistance, we used the criteria of the European Committee on Antimicrobial Susceptibility Testing EUCAST 4.0 (2014) [24]. The presence of beta-lactamase enzyme production, which provides high-level resistance to penicillins, was detected by using the nitrocefin test (BBL DrySlide ${ }^{\mathrm{m}}$, Becton, Dickinson, NJ, USA). Isolates testing positive for beta-lactamase were defined as penicillinase-producing N. gonorrhoeae (PPNG).

The working stock of $N$. gonorrhoeae isolates was stored at $-80{ }^{\circ} \mathrm{C}$. N. gonorrhoeae strains ATCC 49226 and WHO-reference strains G, K, M, O and P were used with each batch of Etest as quality controls [25]. Ceftriaxone and cefixime were tested twice when the MIC was $\geq 0.125 \mathrm{mg} / \mathrm{L}$. Isolates tested in the CL were characterized by their resistance patterns.

An overview of the analysis of isolates tested in CL is given in Table 1. Note that a direct comparison of historical German data with current GORENET data was not possible because different methods for isolate collection were in place before GORENET was rolled out and the geographical coverage was different.

For categorical variables we calculated percentages and, for continuous variables, medians together with interquartile ranges (IQR) were determined. Percentages were compared by Chi-squared or Fisher's exact tests, and medians were compared with the Wilcoxon-MannWhitney test, where applicable. The Kruskal-Wallis test was used to compare continuous variables between more than two categories. Significance level was set at a $p$-value $<0.05$.

\section{Results}

Of the 100 laboratories that were interested in participating in GORENET, 31 were selected for recruitment, and 23 agreed and reported data to GORENET. The reasons for declining participation in GORENET were not using the Etest for $N$. gonorrhoeae susceptibility

Table 1 Isolates tested for susceptibility in network laboratories and in CL, number of laboratories and isolates included in analysis and description of performed analysis for each data source

\begin{tabular}{|c|c|c|c|c|c|c|}
\hline & \multirow{2}{*}{$\begin{array}{l}\text { Number of } \\
\text { laboratories }\end{array}$} & \multicolumn{3}{|c|}{ Number of samples or isolates } & \multirow{2}{*}{$\begin{array}{l}\text { In manuscript } \\
\text { referred as }\end{array}$} & \multirow[t]{2}{*}{ Performed analysis } \\
\hline & & Total & 2014 & 2015 & & \\
\hline $\begin{array}{l}\text { Samples tested for } N \text {. gonorrhoeae } \\
\text { susceptibility in network laboratories }\end{array}$ & 23 & 1654 & 727 & 927 & Samples & $\begin{array}{l}\text { Geographical, age and gender } \\
\text { distribution. Sampled material } \\
\text { and treating specialist }\end{array}$ \\
\hline Isolates tested in $\mathrm{CL}$ & 16 & 537 & 261 & 276 & Isolates & $\begin{array}{l}\text { Susceptibility for ceftriaxone, } \\
\text { cefixime, azithromycin, penicillin } \\
\text { and ciprofloxacin. Presence of } \\
\text { beta-lactamases }\end{array}$ \\
\hline
\end{tabular}


testing $(n=5)$ and too much time and effort needed for participation $(n=3)$.

\section{Continuous routine data collection}

Twenty-three participating laboratories reported to the RKI in total $1654 N$. gonorrhoeae samples tested for susceptibility. Of them, 727 were collected from April to December 2014, and 927 were collected from January to December 2015. Overview of the reported samples is given in Table 1.

The number of reported samples varied between 2 and 305 per laboratory, with a median of 43 samples (IQR 24-86). In total $47.1 \%$ of the samples were reported by three laboratories, which provided information on 209 (in Hamburg), 265 (in Berlin) and 305 (in North RhineWestphalia) samples, respectively.

The number of reported $N$. gonorrhoeae samples varied by administrative district between 1 and 209 (Fig. 1). The three laboratories submitting the majority of the data were located in the areas with $>50$ samples.

Central and northern Germany were represented equally. Data from southern Germany mostly originated from the larger cities.

Overall, $90.0 \%$ of samples tested for susceptibility in network laboratories were from men; $9.5 \%$ were from women and in nine samples, information on gender was not available. The median age of tested men and women was 33 (IQR 25-44) and 27 (IQR 22-40) years respectively $(p$-value $<0.001)$. The distribution of

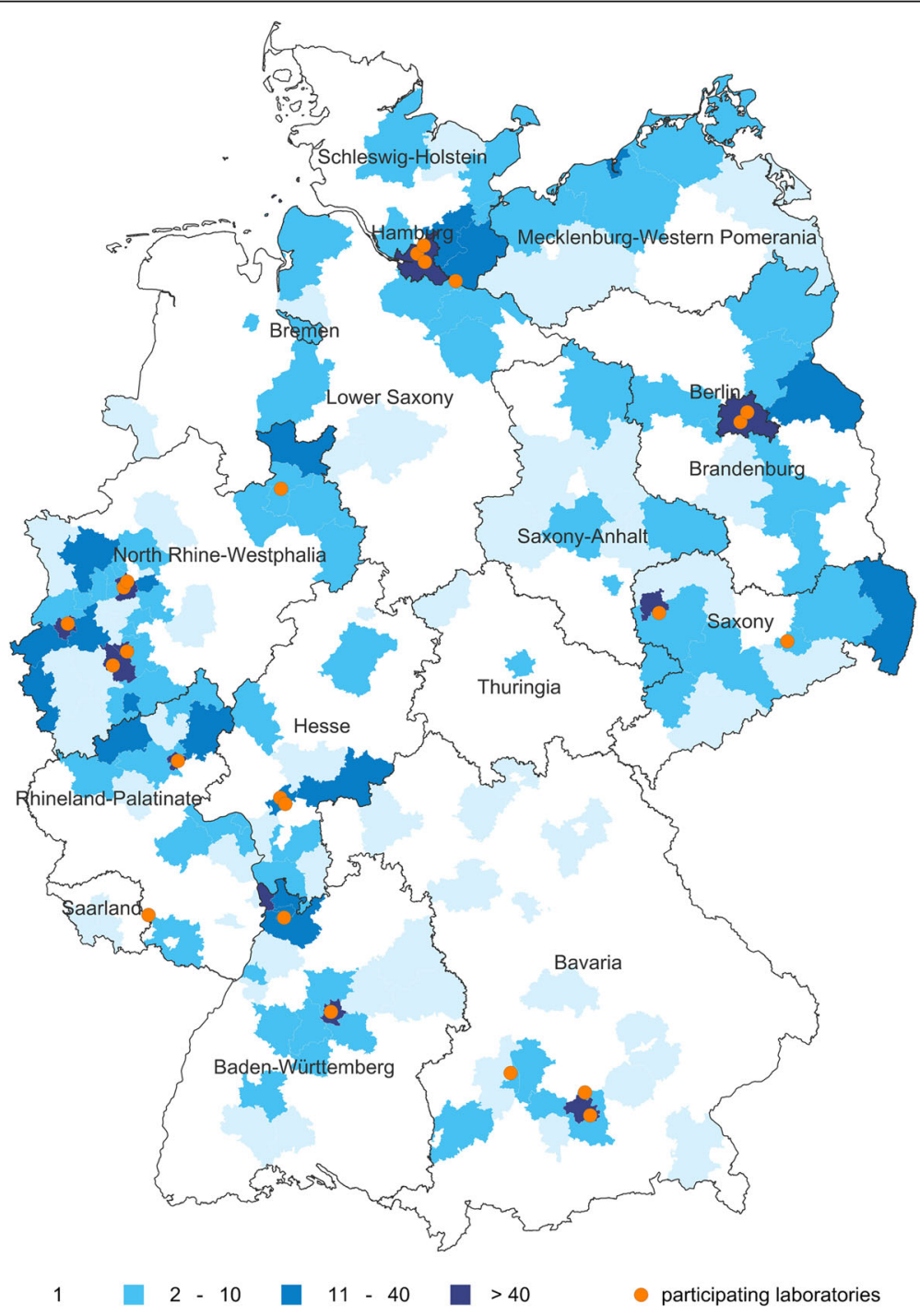

Fig. 1 Distribution of N. gonorrhoeae samples by district in Germany. One thousand, six hundred and fifty-four samples from April 2014 to December 2015 (1366 defined by district code of the patient, 288 defined by district code of the laboratory). Map developed with RegioGraph Software 
$N$. gonorrhoeae susceptibility testing by age group and gender is displayed in Fig. 2.

Most tested samples from men were from urethral (96.1\%) and rectal swabs (1.7\%), while among women samples came from predominately endocervical or vaginal swabs (84.3\%). Most of the samples tested from men were ordered by urologists (74.4\%) and from women by gynaecologists (79.7\%). Distribution by gender $(p$-value $=0.25)$, age $(p$-value $=0.28$ for men and $p$ value $=0.87$ for women $)$, tested material $(p$-value $=0.15$ for men and $\mathrm{p}$-value $=0.07$ for women) and ordering specialist $(p$-value $=0.63$ for men and $p$-value $=0.73$ for women) did not differ between the years 2014 and 2015.

\section{Isolate collection and susceptibility testing}

From the recruited 23 laboratories submitting information on samples tested for $N$. gonorrhoeae susceptibility, 16 sent isolates to the CL. We received 261 viable isolates collected between April and December 2014 and 276 viable isolates collected between January and December 2015. It was determined that $91.4 \%$ of isolates were from men, $8.4 \%$ were from women, and for one of the samples the gender was unspecified. The median age was 33 (IQR 26-43) for isolates from men and 28 (IQR 23-41) for isolates from women. These 537 isolates were tested for susceptibility in the CL. An Overview of the isolates tested in CL is given in Table 1 . The results of the AMR testing of all isolates are summarized in Table 2. The percentage of resistant, intermediate and susceptible isolates did not significantly differ by age or gender.

No resistance to ceftriaxone (MIC $>0.125 \mathrm{mg} / \mathrm{L}$ ) was detected in 2014 or 2015 (Fig. 3). In 2014, two isolates showed MICs at the estimated breakpoint of $0.125 \mathrm{mg} /$ L. One of these isolates displayed further resistance to cefixime, azithromycin and ciprofloxacin and showed an intermediate test result to penicillin (isolate 1, Table 3).
Another isolate displayed resistance to ciprofloxacin and penicillin, while it was intermediate to azithromycin and had a MIC value at the breakpoint for cefixime (isolate 10, Table 3).

Altogether, 1.9\% $(n=5)$ in 2014 and $1.4 \%(n=4)$ in 2015 of the isolates displayed resistance (MIC $>0.125 \mathrm{mg} / \mathrm{L}$ ) to cefixime. The majority of isolates $(62.5 \%$ in 2014 and $77.9 \%$ in 2015) showed low MICs of $\leq 0.016 \mathrm{mg} / \mathrm{L}$ to cefixime (Fig. 4). In 2014, 3.8\% $(n=10)$ and in 2015, $1.4 \%(n=4)$ of the isolates had a MIC of $0.125 \mathrm{mg} / \mathrm{L}$ at the estimated breakpoint.

All isolates with resistance to cefixime displayed resistance to ciprofloxacin. Three of the nine cefiximeresistant strains also showed resistance to azithromycin. No cefixime-resistant strain was susceptible to penicillin (3 resistant, 6 intermediate).

One cefixime-resistant isolate (MIC $0.25 \mathrm{mg} / \mathrm{L}$ ) displayed additional resistance against azithromycin and ciprofloxacin, intermediate susceptibility against penicillin and showed reduced susceptibility to ceftriaxone at the breakpoint of MIC $0.125 \mathrm{mg} / \mathrm{L}$ (isolate 1, Table 3).

All isolates displaying resistance to cefixime were from men.

A total of $11.9 \%$ (2014) and 9.8\% (2015) of the isolates showed resistance against azithromycin (MIC $>0.5 \mathrm{mg} / \mathrm{L}$ ). In addition, there was a high percentage of $N$. gonorrhoeae strains with intermediate susceptibility (33.7\% in 2014 and $28.3 \%$ in 2015) and the MIC distribution of the susceptible strains appeared closer to intermediate breakpoint (MIC $>0.38 \mathrm{mg} / \mathrm{L}$ ) (Fig. 5). The MICs of resistant strains were mostly low and showed a distribution concentrating around the breakpoint (MIC $>0.5 \mathrm{mg} / \mathrm{L}$ ).

In 2015, one isolate displayed high-level resistance to azithromycin (MIC $>256 \mathrm{mg} / \mathrm{L}$ ). This isolate also showed high-level resistance to penicillin and resistance to ciprofloxacin, but was susceptible to ceftriaxone and cefixime.

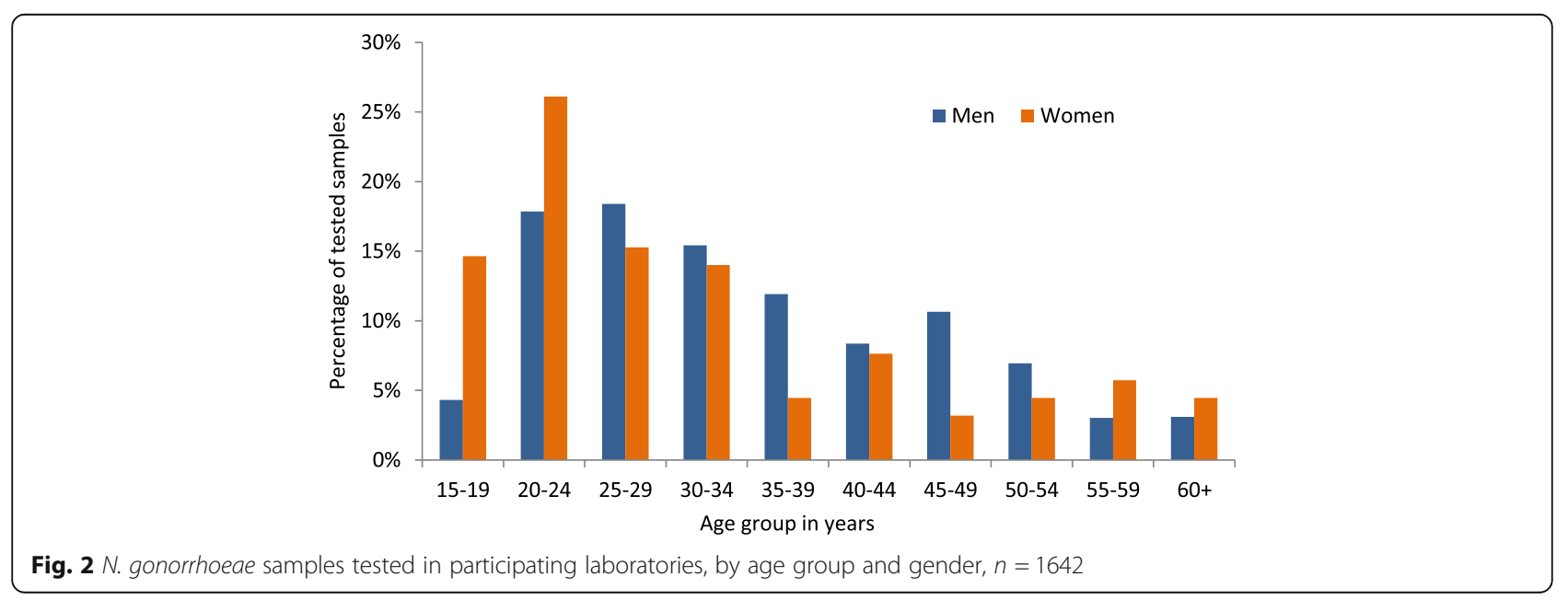


Table 2 Number and percentage of $\mathrm{N}$. gonorrhoeae isolates testing susceptible, intermediate and resistant against cefixime, ceftriaxone, azithromycin, penicillin and ciprofloxacin, $n=261$ for year 2014 and $n=276$ for year 2015, ${ }^{*} p$-value $<0.05$

\begin{tabular}{|c|c|c|c|c|c|c|}
\hline & Susceptible & & Intermedia & & Resistant & \\
\hline & Number ( $p$ & & Number ( $p$ & & Number ( $p$ & \\
\hline & 2014 & 2015 & 2014 & 2015 & 2014 & 2015 \\
\hline Cefixime & $256(98.1)$ & $272(98.6)$ & l & / & $5(1.9)$ & $4(1.4)$ \\
\hline Ceftriaxone & $261(100)$ & $276(100)$ & / & / & $0(0)$ & $0(0)$ \\
\hline Azithromycin & $142(54.4)$ & $171(62.0)$ & $88(33.7)$ & $78(28.3)$ & $31(11.9)$ & $27(9.8)$ \\
\hline Ciprofloxacin* & $73(28.0)$ & $114(41.3)$ & $0(0)$ & $1(0.4)$ & $188(72.0)$ & $161(58.3)$ \\
\hline Penicillin* & $27(10.3)$ & $40(14.5)$ & $158(60.5)$ & $184(66.7)$ & $76(29.1)$ & $52(18.8)$ \\
\hline
\end{tabular}

The percentage of strains with resistance to ciprofloxacin $(\mathrm{MIC}>0.06 \mathrm{mg} / \mathrm{L}$ ) was $72.0 \%$ in 2014 and $58.3 \%$ in 2015 (Table 2).

Overall, $29.1 \%$ of the isolates in 2014 and $18.8 \%$ in 2015 displayed resistance to penicillin. In addition, there was a very high rate of intermediate $N$. gonorrhoeae strains (Table 2).

Nitrocefin testing for the detection of beta-lactamase activity in $N$. gonorrhoeae was performed in 83.5\% $(n=218)$ of isolates in 2014. All 276 isolates $(100 \%)$ were tested for beta-lactamase activity in 2015. Highlevel plasmid-mediated resistance against penicillin (penicillinase producing $N$. gonorrhoeae, PPNG) was found in 25\% of all tested strains in 2014 and in 14\% of the strains in 2015 .

\section{Discussion}

Using GORENET we aimed at two targets concerning gonococcal infections: data collection on disease epidemiology and monitoring of resistance patterns with unified methodology.

We were able to implement a nationwide data collection of all performed $N$. gonorrhoeae susceptibility testing in the participating laboratories. Routine data collection on all performed $N$. gonorrhoeae susceptibility tests together with epidemiological information, like age and gender, was not in place until GORENET surveillance.

Before starting GORENET, the CL collected isolates from a range of laboratories. This pre-existing network was expanded within GORENET, and the number of collected isolates increased substantially.

We were able to set up electronic data collection for all samples tested for $N$. gonorrhoeae susceptibility in the participating laboratories. Timely transmitted data are a good tool for monitoring $N$. gonorrhoeae susceptibility dynamics. Due to data protection issues, the collected epidemiological information was limited and important information, such as data regarding the transmission route, therapeutic regimen and therapeutic success, could not be gathered.

We reached a relatively even geographical representation of all regions, but the coverage in central and southern Germany should be increased further.

In routine data collection from the network laboratories, we found that over $90 \%$ of all samples tested for $N$. gonorrhoeae susceptibility were from men, similar to several other European countries [22]. This percentage

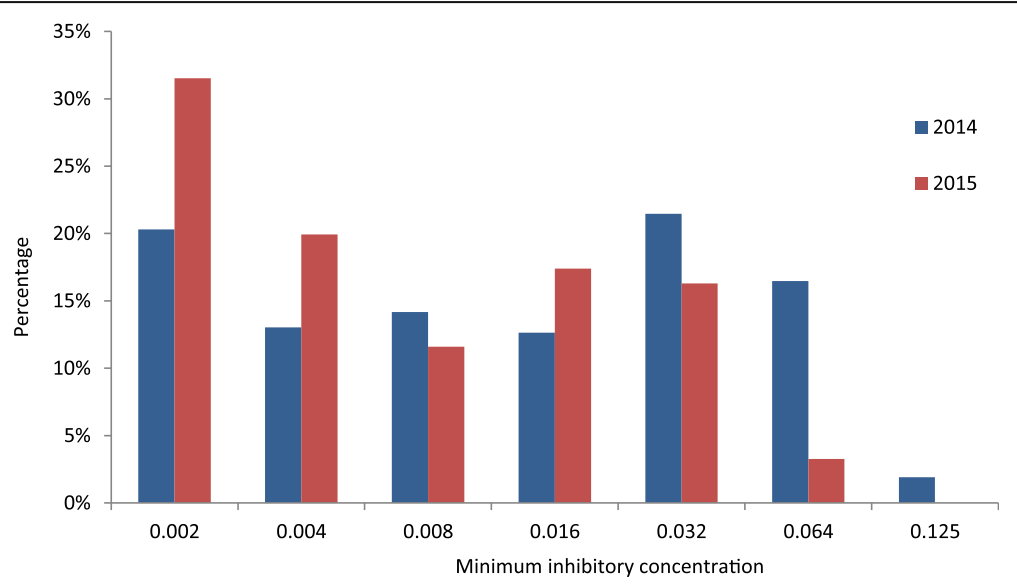

Fig. 3 Distribution of minimum inhibitory concentrations for ceftriaxone, $n=261$ for year 2014 and $n=276$ for year 2015 
Table 3 Minimum inhibitory concentrations (MIC) of cefixime, ceftriaxone, azithromycin, ciprofloxacin and penicillin for isolates with resistance to cefixime or with MICs at the breakpoint for resistance to cefixime $(n=24)$

\begin{tabular}{|c|c|c|c|c|c|}
\hline \multirow[b]{2}{*}{ Isolate } & \multicolumn{5}{|c|}{ Minimum inhibitory concentration (MIC) } \\
\hline & Cefixime & Ceftriaxone & Azithromycin & Ciprofloxacin & Penicillin \\
\hline 1 & 0.25 & 0.125 & 0.75 & 32 & 1 \\
\hline 2 & 0.19 & 0.047 & 1 & 0.25 & 32 \\
\hline 3 & 0.19 & 0.094 & 0.75 & 32 & 3 \\
\hline 4 & 0.19 & 0.023 & 0.19 & 32 & 0.75 \\
\hline 5 & 0.19 & 0.047 & 0.125 & 32 & 0.75 \\
\hline 6 & 0.19 & 0.032 & 0.19 & 32 & 1.5 \\
\hline 7 & 0.19 & 0.032 & 0.094 & 12 & 0.75 \\
\hline 8 & 0.19 & 0.032 & 0.19 & 16 & 1 \\
\hline 9 & 0.19 & 0.023 & 0.125 & 16 & 0.75 \\
\hline 10 & 0.125 & 0.125 & 0.5 & 32 & 2 \\
\hline 11 & 0.125 & 0.064 & 1 & 32 & 1 \\
\hline 12 & 0.125 & 0.064 & 1 & 32 & 2 \\
\hline 13 & 0.125 & 0.032 & 0.19 & 32 & 0.75 \\
\hline 14 & 0.125 & 0.032 & 0.19 & 32 & 0.75 \\
\hline 15 & 0.125 & 0.032 & 0.19 & 32 & 0.5 \\
\hline 16 & 0.125 & 0.032 & 0.125 & 32 & 1.5 \\
\hline 17 & 0.125 & 0.023 & 0.125 & 32 & 0.38 \\
\hline 18 & 0.125 & 0.023 & 0.19 & 32 & 0.75 \\
\hline 19 & 0.125 & 0.023 & 0.19 & 32 & 0.5 \\
\hline 20 & 0.125 & 0.023 & 0.125 & 16 & 0.75 \\
\hline 21 & 0.125 & 0.023 & 0.094 & 12 & 0.75 \\
\hline 22 & 0.125 & 0.016 & 0.25 & 32 & 0.75 \\
\hline 23 & 0.125 & 0.012 & 0.125 & 12 & 0.38 \\
\hline 24 & 0.016 & 0.006 & 256 & 12 & 32 \\
\hline
\end{tabular}

was $84 \%$ in previous studies in Germany [15], and we can assume that at least half of all isolates from men are attributable to men having sex with men (MSM), comparable to other European countries [22]. Nevertheless, women might still be underrepresented in our sample. This seems possible because men are more often symptomatic and might therefore be tested more often than women [14]. We aim to collect data on transmission routes from physicians to better interpret the collected data.

We also found that the median age of tested men was slightly higher than that of tested women. Again, EuroGASP data and other previous data from Germany have reported findings $[18,21,22]$ similar to our results. In countries that reported the risk of transmission, the proportion of men aged $>25$ years was higher among MSM than among heterosexual men. This might explain the higher median age of the tested men in our sample. As $N$. gonorrhoeae is not reportable in Germany, we were not able to compare whether the $N$. gonorrhoeae AMR test distribution by age corresponded to the age groups most affected by gonorrhoea.

We exclusively analysed susceptibility data from the $\mathrm{CL}$ testing because presently Germany lacks a standard operation protocol and regular quality assurance for N. gonorrhoeae testing for all laboratories. From a cross-sectional survey among laboratories performing $N$. gonorrhoeae diagnostics we know that $55 \%$ of all laboratories (76\% of private laboratories) are accredited and $19 \%$ are certified [23]. However, due to the use of different methods, standards and test panels it is unknown if there are substantial differences in quality of testing, and GORENET will be a useful tool for quality assurance in the future.

Susceptibility testing in the CL enabled the monitoring of $N$. gonorrhoeae antimicrobial resistance detected by a unified methodology. Age and gender distribution of the

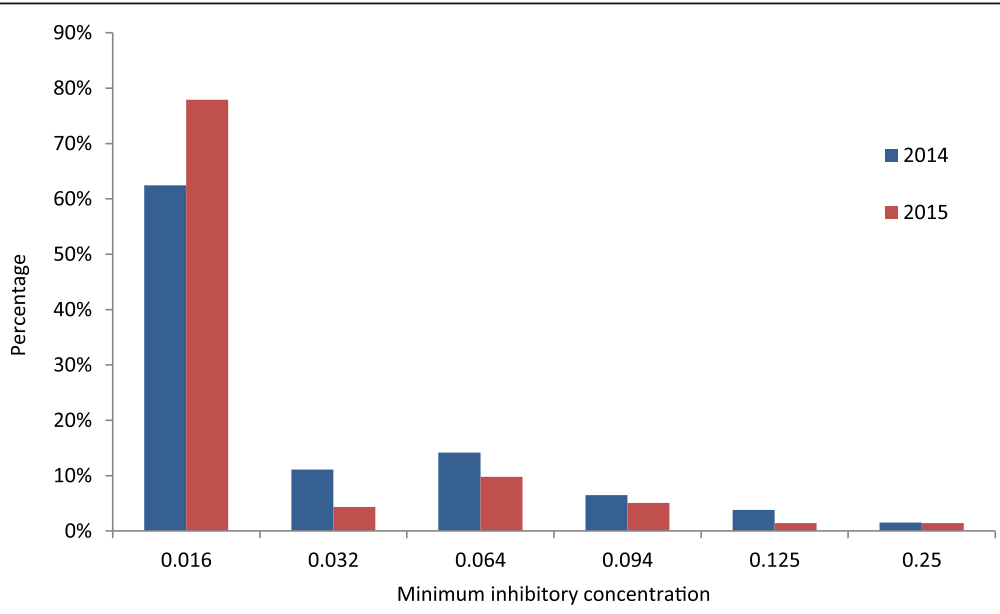

Fig. 4 Distribution of minimum inhibitory concentration for cefixime, $n=261$ for year 2014 and $n=276$ for year 2015 


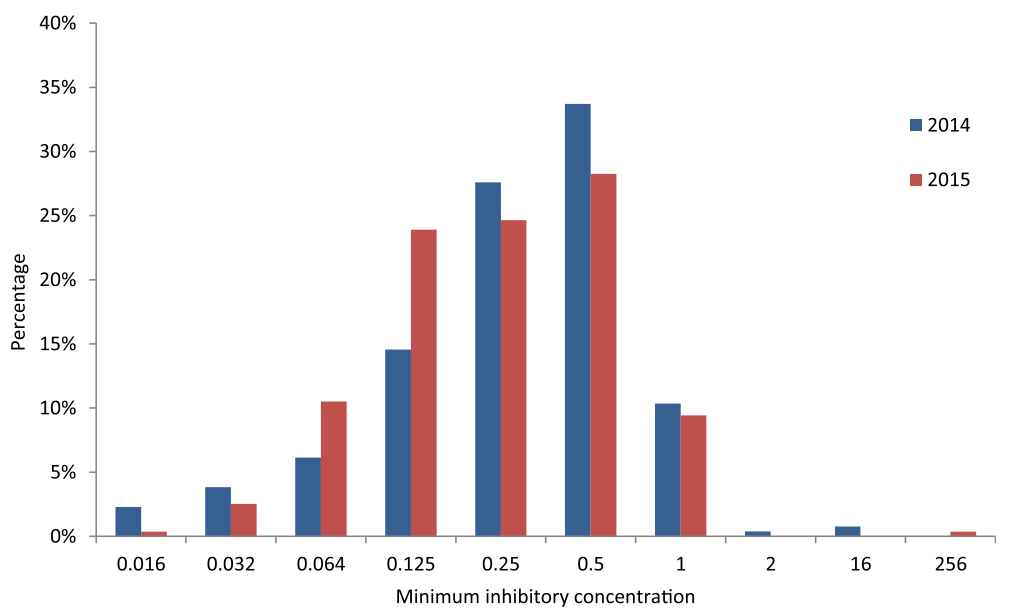

Fig. 5 Distribution of minimum inhibitory concentration for azithromycin, $n=261$ for year 2014 and $n=276$ for year 2015

tested isolates did not differ from all samples tested for susceptibility in the participating laboratories.

No resistance to ceftriaxone was detected in 2014 and 2015 in isolates collected with GORENET, and only two isolates showed MICs at the estimated breakpoint of $0.125 \mathrm{mg} / \mathrm{L}$.

Previous German publications also did not show notable levels of ceftriaxone resistance or ESC resistance [18-21] in Germany. However, within the Euro-GASP collection, there was one exception in 2011, when 6.5\% of German strains were resistant against ceftriaxone. In the following years 2012-2014, there were no notable levels of ceftriaxone resistance observed [18, 21, 22] in Germany by the Euro-GASP surveillance.

Currently, parenterally administered ceftriaxone remains an effective treatment option for gonorrhoea in Germany. Similar data have been published from surveillance systems in other countries $[13,26]$.

The percentage of strains with resistance to cefixime remained moderate within the GORENET $(<2 \%)$, and low MIC values were predominant. German Euro-GASP data from 2014 correspond to this GORENET observation. In 2014 no cefixime-resistant isolate from Germany was detected within the Euro-GASP surveillance [22]. Compared with previous German data from Euro-GASP, this is a decrease from the years 2009-2013, when resistance rates from $5.7 \%$ to $12.9 \%$ were detected [22]. The decreasing number of strains resistant to cefixime could be observed not only in GORENET: European Euro-GASP data from 2014 and surveillance reports from the United Kingdom, the United States and Canada showed the same trend to less ESC-resistant isolates [26-29]. The decrease in gonococcal resistance to cefixime in 2014/15 suggests that clinicians in Germany may have avoided prescribing this antibiotic as a first-line treatment after the new therapy guidelines were published. However, changes in resistance patterns develop incrementally and are usually not detected so fast.

A presumptive explanation for this observation is the eradication of previously undetected reservoirs [27, 30]. Especially extragenital infections, which are oftentimes asymptomatic, difficult to culture and difficult to treat, are a constant reservoir for the spread of gonorrhoea $[9,30,31]$. Due to regular use of highly sensitive molecular diagnostic tests, like nucleic acid amplification tests (NAAT), in the routine diagnostic, detection of gonococcal infections was improved. Extragenital infections and subclinical urogenital infections are therefore more often confirmed and can be successfully treated [31]. The adjustment of treatment guidelines for ceftriaxone as first-line therapy seems to provide here an additional benefit. Sufficient treatment of these infections prevents a selection of resistant clones and might be a reason for the decrease of cefixime-resistant gonococci [27, 28, 30].

Molecular typing studies, performed with $N$. gonorrhoeae multiantigen sequence typing (NG-MAST, http://www.ngmast.net) [32], showed one sequence type (ST1407), which was the most frequently observed sequence type associated with ESC- and multi-resistance [33-35]. Therefore, a further reason for the decrease of cefixime resistance could be a replacement of this multidrug-resistant $N$. gonorrhoeae clone ST1407 by clones with different resistance patterns within the infected population [27]. This could be an effect of the sufficient treatment or, as the Euro-GASP authors pointed out, be caused by impaired reinfection with the same clone due to a partial immunity and needs to be evaluated by future typing studies [27]. Additional monitoring and a molecular typing study within GORENET in the next years are therefore intended.

Multidrug resistance was not detected regularly in GORENET. Only one cefixime-resistant isolate displayed further resistance to azithromycin and ciprofloxacin, 
intermediate susceptibility to penicillin and a reduced susceptibility to ceftriaxone at the breakpoint MIC of $0.125 \mathrm{mg} / \mathrm{L}$. Nevertheless, the combination of resistances is particularly alarming and should be monitored further.

A high prevalence of resistance was detected for azithromycin. Although we observed mostly resistance near the breakpoint (MIC $>0.5 \mathrm{mg} / \mathrm{L}$ ), this trend is concerning. In addition, there was a high rate of intermediate $N$. gonorrhoeae strains: $40-45 \%$ of the strains were not fully susceptible to azithromycin. Germany's data from previous years [22] shows that the level of azithromycin resistance was mostly under $5 \%$. The first-line use of azithromycin is very common in STI treatment regimes, especially as a syndromic treatment before or without confirmation of the pathogenic agent. This might explain the increase in azithromycin resistance in the last several years, but data on prescriptions in Germany are not published.

In 2015, we detected the first case of a high-level azithromycin resistant $N$. gonorrhoeae strain in Germany, with a MIC $>256 \mathrm{mg} / \mathrm{L}$. This isolate was susceptible to ceftriaxone and cefixime but showed high-level resistance to penicillin and resistance to ciprofloxacin. As rising resistance rates to azithromycin are increasingly observed globally $[22,26]$ and high-level azithromycin resistance is reported worldwide [35-43], azithromycin is not suitable for first-line treatment. If azithromycin is used as a single-drug treatment in cases of severe penicillin/cephalosporin anaphylaxis, susceptibility testing prior to treatment should be performed.

According to current treatment guidelines, dual therapy with ceftriaxone and azithromycin is recommended, using two antimicrobial agents with different mechanisms of action [5, 44]. Currently, there is no alternative to this dual treatment regime. Ultimately, we will need to discuss whether we have to abandon dual therapy with azithromycin if the trend of increasing azithromycin resistance remains in the years to come $[9,45]$.

The resistance rates to ciprofloxacin were constantly high in Germany, although a drop was detectable in the surveillance period. A high prevalence of resistance to ciprofloxacin has also been found in Europe and worldwide since the late 1990s [22]. The drug is therefore not recommended for therapeutic use in Germany.

Resistance to penicillin has been prevalent for many decades worldwide. Nearly $25 \%$ of all isolates displayed resistance to penicillin and an additional $64 \%$ showed intermediate susceptibility in the GORENET surveillance data. Accordingly, approximately $90 \%$ of the strains were not fully susceptible to penicillin in Germany. High-level plasmid-mediated resistance to penicillin was also regularly observed but decreased within the surveillance period from $25.9 \%$ to $14 \%$, an observation that requires further monitoring.
We have several limitations in our data. First, within the GORENET data collection scheme, we were unable to collect more detailed epidemiological information, such as the risk of transmission, special symptoms, therapy strategies and treatment success rates. Usually, laboratories in Germany have very limited epidemiological information available, provided by the physicians in charge, and need to treat these data confidentially due to strict data-protection regulations.

Second, our data analysis was limited by the small number of isolates collected compared to the overall population of Germany.

Third, we cannot exclude selection bias in our data, because we recruited laboratories based on their catchment area, number of tested $N$. gonorrhoeae samples and use of the Etest from a pool of laboratories that expressed an interest to participate. However, with selected and established network laboratories, which forwarded all of their received isolates, we diminished a collection bias from laboratories being more prone to submit isolates with interesting resistance patterns.

\section{Conclusions}

Using GORENET, we were able to implement a nationwide collection of all performed $N$. gonorrhoeae susceptibility data in the participating laboratories and increase the number of collected isolates retested at the CL for confirmation and quality assurance. The majority of susceptibility tests are performed among young men. More detailed epidemiological information would be beneficial.

The resistance rate to ceftriaxone remains low in Germany. Therefore, ceftriaxone is still an appropriate treatment for gonorrhoea at present. In 2014 and 2015, we found low resistance rates for cefixime in Germany. However, this needs further monitoring. Resistance to azithromycin is common and should continue to be monitored in the future. Except for a small decrease in AMR towards ciprofloxacin and penicillin, no substantial changes in the susceptibility patterns between 2014 and 2015 could be detected.

In conclusion, GORENET as a gonococcal antimicrobial surveillance in Germany is highly needed. Current data and ongoing collection of data will be used to update national treatment guidelines and, if necessary, implementation of future prevention measures.

To continue to monitor $N$. gonorrhoeae susceptibility, particularly against ESC and azithromycin, the yearly number of isolates tested in the CL should be substantially increased. Molecular surveillance of the circulating strains is important for monitoring the current situation, the evolving resistances and the transmission networks.

Surveillance of susceptibility is essential to ensure efficient patient management and keep gonorrhoea a treatable disease. 


\section{Abbreviations}

AMR: Antimicrobial resistance; ARS: Antibiotic resistance surveillance programme; ATCC: American Type Culture Collection; CDC: Centers for Disease Control and Prevention; CL: Consiliary Laboratory for gonococci; ECDC: European Centre for Disease Prevention and Control; ESC: Extended spectrum cephalosporin; EUCAST: European Committee on Antimicrobial Susceptibility Testing; Euro-GASP: European Gonococcal Antimicrobial Surveillance Programme; GORENET: Gonococcal Resistance Network; i.m.: Intramuscular; i.v.: Intravenous; IQR: Interquartile ranges; MSM: Men having sex with men; N gonorrhoeae: Neisseria gonorrhoeae; NAAT: nucleic acid amplification test; NG MAST: Neisseria gonorrhoeae multiantigen sequence typing; PPNG: Penicillinase producing N. gonorrhoeae; RKI: Robert Koch Institute; ST: Sequence type; STI: Sexually transmitted infection; WHO: World Health Organization

\section{Acknowledgements \\ *GORENET study group.}

Thomas Back (Labor Schottdorf MVZ GmbH, Augsburg).

Anja Berger (Bayerisches Landesamt für Gesundheit und

Lebensmittelsicherheit, Oberschleißheim, Sachgebiet GE 2.2 Infektiologie,

Humanbakteriologie, Mykologie und Konsiliarlabor Diphtherie).

Valerie Chapot, Jörg Steinmann (Institut für Medizinische Mikrobiologie,

Universitätsklinikum Essen).

Stephan Eicke (Labor Dr. Eicke, Berlin).

Claudia Friedrichs (Medizinisches Labor Ostsachsen MVZ GbR, Görlitz).

Andreas Groß, Hans-Jochen Hagedorn (Labor Krone, Bad Salzuflen).

Alexander Halfmann (Institut für Medizinische Mikrobiologie und Hygiene,

Universitätsklinikum des Saarlandes, Homburg).

Britt Hornei (MVZ Synlab Leverkusen GmbH, Leverkusen).

Ralf Ignatius (Labor Enders und Kollegen MVZ, Stuttgart).

Simone Korten, Hany Sahly (Labor Lademannbogen MVZ GmbH, Hamburg).

Elzbieta Kozub-Witkowski (LADR GmbH Medizinisches Versorgungszentrum

Dr. Kramer \& Kollegen, Geesthacht).

Sabine Krämer (MVZ Labor Eveld \& Kollegen, Essen).

Margit Kühn (Labor Becker, Olgemöller und Kollegen, München).

Anke Liebetrau (MVZ Labor Dr. Reising-Ackermann und Kollegen, Leipzig).

Thomas Meyer (Institut für Medizinische Mikrobiologie, Virologie und

Hygiene, Universitätsklinikum Hamburg-Eppendorf)

Klaus Oberdorfer (Labor Dr. Limbach und Kollegen, Heidelberg).

Roland Pfüller (MDI Laboratorien GmbH, Berlin).

Caroline Ruckert (MVZ für Laboratoriumsmedizin und Mikrobiologie KoblenzMittelrhein, Koblenz).

Roman Schwarz (Labor Mönchengladbach, Medizinisches

Versorgungszentrum Dr. Stein + Kollegen, Limbach Gruppe).

Daniela Walch, Madeleine Mai (Institut für Labormedizin, Mikrobiologie und

Krankenhaushygiene, Krankenhaus Nordwest, Frankfurt am Main).

Thomas A. Wichelhaus (Institut für Medizinische Mikrobiologie und

Krankenhaushygiene, Universitätsklinikum Frankfurt, Goethe-Universität).

Hilmar Wisplinghoff (Labor Dr. Wisplinghoff, Köln).

Nicole Wüppenhorst (Institut für Hygiene und Umwelt, Abteilung

Medizinische Mikrobiologie, Hamburg)

We acknowledge further persons for their work and involvement:

Lorenz Jäger, Elzbieta Nowakowski, Elisabeth Schlosser, Tanja Sinninger und

Doris Streit-Schmid from Labor Schottdorf MVZ GmbH, Augsburg.

Annegret Mahl from Bayerisches Landesamt für Gesundheit und

Lebensmittelsicherheit, Oberschleißheim.

Doris Kahle from Medizinisches Labor Ostsachsen MVZ GbR, Görlitz.

Michael Zimmer, Olga Zimmer and Vanessa Dreyer from Labor Krone, Bad

Salzuflen.

Thomas Regnath from Labor Enders und Kollegen MVZ, Stuttgart.

Bettina Hebold from MVZ Labor Dr. Reising-Ackermann und Kollegen, Leipzig.

Beatrice Weber from Institut für Medizinische Mikrobiologie, Virologie und

Hygiene, Universitätsklinikum Hamburg-Eppendorf.

Mirijam Schlicht, Antje Mewes and Michael Friedrich from MDI Laboratorien

$\mathrm{GmbH}$, Berlin.

Denia Frank from Institut für Medizinische Mikrobiologie und

Krankenhaushygiene, Universitätsklinikum Frankfurt, Goethe-Universität.

Kerstin Dehmel from Robert Koch Institute.

\section{Funding}

The project was designed by Robert Koch Institute and Consiliary Laboratory for Gonococci. The project was funded by German Federal Ministry of Health.
German Federal Ministry of Health was not involved in data collection, analysis and writing of the manuscript.

\section{Availability of data and materials}

The data sets generated and analysed during the current study are available in the ZENODO.

(https://doi.org/10.5281/zenodo.437583).

\section{Authors' contributions}

SB, SD, PKK and VB developed the concept and methods of the Gonococcal Resistance Network (GORENET). SB, SD, KJ, AL, AS, SN, PKK and VB were responsible for implementation of the GORENET. SB, EG and PKK were responsible for laboratory testing. SD, AS, KJ, SN and VB were in charge of the data collection and analysis. SB and SD drafted the paper and share the first authorship in equal parts. All authors revised the paper. All authors read and approved the final manuscript.

\section{Ethics approval and consent to participate}

The data-collection protocol was confirmed by the data-protection officer. Additional approval from an ethics committee was deemed not necessary, as no patient-identifying data were collected. According to the German Data Protection Act the study complies with the national guidelines, and no formal ethical committee approval was necessary (available under https://www.gesetze-im-internet.de/bdsg_1990/index.html)

\section{Consent for publication}

Not applicable

\section{Competing interests}

Klaus Jansen is a member of the editorial board (Associate Editor) of this journal. All other authors declare that they have no competing interests.

\section{Publisher's Note}

Springer Nature remains neutral with regard to jurisdictional claims in published maps and institutional affiliations.

\section{Author details}

${ }^{1}$ German Consiliary Laboratory for Gonococci, Department of Dermatology and Venerology, Vivantes Hospital Berlin, Berlin, Germany. ${ }^{2}$ Department for Infectious Disease Epidemiology, Robert Koch Institute, Berlin, Germany. ${ }^{3}$ Charité University Medicine Berlin, Berlin, Germany. ${ }^{4}$ Department of Infectious Diseases, Robert Koch Institute, Berlin, Germany. ${ }^{5}$ European Public Health Microbiology Training (EUPHEM) programme, European Centre for Disease Prevention and Control (ECDC), Stockholm, Sweden.

Received: 23 March 2017 Accepted: 3 January 2018

Published online: 17 January 2018

\section{References}

1. WHO. Global action plan to control the spread and impact of antimicrobial resistance in Neisseria gonorrhoeae. World Health Organization, Department of Reproductive Health and Research; 2012. http://apps.who.int/iris/bitstream/ 10665/44863/1/9789241503501_eng.pdf.

2. Centers for Disease C, Prevention. CDC grand rounds: the growing threat of multidrug-resistant gonorrhea. MMWR Morb Mortal Wkly Rep. 2013;62(6):103-6.

3. European Centre for Disease Prevention and Control. Response plan to control and manage the threat of multidrug-resistant gonorrhoea in Europe. Stockholm: European Centre for Disease Prevention and Control, 2012; 2012. Report No

4. Bignell CJ. European guideline for the management of gonorrhoea. Int J STD AIDS. 2001;12(Suppl 3):27-9.

5. Unemo M, Shafer WM. Antimicrobial resistance in Neisseria gonorrhoeae in the 21st century: past, evolution, and future. Clin Microbiol Rev. 2014;27(3):587-613.

6. Unemo M, Shafer WM. Antibiotic resistance in Neisseria gonorrhoeae: origin, evolution, and lessons learned for the future. Ann N Y Acad Sci. 2011;1230:E19-28.

7. Bolan GA, Sparling PF, Wasserheit JN. The emerging threat of untreatable gonococcal infection. N Engl J Med. 2012;366(6):485-7.

8. Unemo M, Nicholas RA. Emergence of multidrug-resistant, extensively drugresistant and untreatable gonorrhea. Future Microbiol. 2012;7(12):1401-22. 
9. Unemo M. Current and future antimicrobial treatment of gonorrhoea - the rapidly evolving Neisseria gonorrhoeae continues to challenge. BMC Infect Dis. 2015;15:364

10. Whiley DM, Goire N, Lahra MM, Donovan B, Limnios AE, Nissen MD, et al. The ticking time bomb: escalating antibiotic resistance in Neisseria gonorrhoeae is a public health disaster in waiting. J Antimicrob Chemother. 2012;67(9):2059-61.

11. Brockmeyer N, Spornraft-Ragaller P, Bremer V, et al. S2k-Leitlinie: Gonorrhoe bei Erwachsenen und Adoleszenten; 2013. http://www.awmf.org/uploads/ tx_szleitlinien/059-004l_S25_Gonorrhoe_bei_Erwachsenen_Adoleszenten_ 2014-verlaengert_01.pdf.

12. Mlynarczyk-Bonikowska B, Serwin AB, Golparian D. Walter de Walthoffen S, Majewski S, Koper M, et al. antimicrobial susceptibility/resistance and genetic characteristics of Neisseria gonorrhoeae isolates from Poland, 20102012. BMC Infect Dis. 2014;14:65.

13. La Ruche G, Goubard A, Berçot B, Cambau E, Semaille C, Sednaoui P. Gonococcal infections and emergence of gonococcal decreased susceptibility tocephalosporins in France, 2001 to 2012. Euro Surveill. 2014; 19(34). https://doi.org/10.2807/1560-7917.ES2014.19.34.20885.

14. Bignell C, Unemo M, European STIGEB. 2012 European guideline on the diagnosis and treatment of gonorrhoea in adults. Int J STD AIDS. 2013;24(2):85-92.

15. Bignell C, FitzGerald M. UK national guideline for the management of gonorrhoea in adults, 2011. Int J STD AIDS. 2011;22(10):541-7.

16. Workowski KA. Centers for disease control and prevention sexually transmitted diseases treatment guidelines. Clin Infect Dis. 2015;61(Suppl 8):S759-62.

17. DSTIG. Gonorrhoe bei Erwachsenen und Adoleszenten. Deutsche STIGesellschaft e. V., 2013.

18. Horn NN, Kresken M, Korber-Irrgang B, Gottig S, Wichelhaus C, Wichelhaus TA, et al. Antimicrobial susceptibility and molecular epidemiology of Neisseria gonorrhoeae in Germany. Int J Med Microbiol. 2014;304(5-6):586-91.

19. Enders M, Turnwald-Maschler A, Regnath T. Antimicrobial resistance of Neisseria gonorrhoeae isolates from the Stuttgart and Heidelberg areas of southern Germany. Eur J Clin Microbiol Infect Dis. 2006;25(5):318-22.

20. Wagner J, Tebbe B, Hornle R, Chahin M, Arvand M, Wendt C, et al. Antibiotic susceptibility of Neisseria gonorrhoeae isolates in Berlin. Hautarzt. 2000;51(9):666-9.

21. Regnath Thomas, Mertes Thomas, Ignatius Ralf. Antimicrobial resistance of Neisseria gonorrhoeae isolates in south-west Germany, 2004 to 2015: increasing minimal inhibitory concentrations of tetracycline but no resistance to third-generation cephalosporins. Euro Surveill. 2016;21(36). https://doi.org/10.2807/1560-7917.ES.2016.21.36.30335.

22. European Centre for Disease Prevention and Control. Gonococcal antimicrobial susceptibility surveillance in Europe 2015. Stockholm: ECDC; 2017. https://ecdc.europa.eu/sites/portal/files/documents/gonococcalantimicrobial-susceptibility-surveillance-Europe-2015.pdf.

23. Loenenbach A, Dudareva-Vizule S, Buder S, Sailer A, Kohl PK, Bremer V. Laboratory practices: diagnostics and antibiotics resistance testing of Neisseria gonorrhoeae in Germany. Bundesgesundheitsblatt Gesundheitsforschung Gesundheitsschutz. 2015:58(8):866-74.

24. European Committee on Antimicrobial Susceptibility Testing. Breakpoint tables for interpretation of MICs and zone diameters. Version 4.0. EUCAST 2014. Available from: http://www.eucast.org/clinical_breakpoints/.

25. Unemo M, Fasth O, Fredlund H, Limnios A, Tapsall J. Phenotypic and genetic characterization of the 2008 WHO Neisseria gonorrhoeae reference strain panel intended for global quality assurance and quality control of gonococcal antimicrobial resistance surveillance for public health purposes. J Antimicrob Chemother. 2009;63(6):1142-51.

26. Martin I, Sawatzky P, Liu G, Allen V, Lefebvre B, Hoang L, et al. Decline in decreased cephalosporin susceptibility and increase in Azithromycin resistance in Neisseria gonorrhoeae. Canada Emerg Infect Dis. 2016;22(1):65-7.

27. Cole MJ, Spiteri G, Jacobsson S, Pitt R, Grigorjev V, Unemo M, et al. Is the tide turning again for cephalosporin resistance in Neisseria gonorrhoeae in Europe? Results from the 2013 European surveillance. BMC Infect Dis. 2015;15:321.

28. Public Health England. GRASP 2013 report: the Gonococcal Resistance to Antimicrobial Surveillance Programme (England and Wales). Public Health England; 2014. https://www.gov.uk/government/uploads/system/uploads/ attachment_data/file/368477/GRASP_Report_2013.pdf.

29. Centers for Disease Control and Prevention. Sexually transmitted diseases surveillance 2014. Centers for Disease Control and Prevention; 2015. https:// www.cdc.gov/std/stats14/surv-2014-print.pdf.
30. Lewis DA. Will targeting oropharyngeal gonorrhoea delay the further emergence of drug-resistant Neisseria gonorrhoeae strains? Sex Transm Infect. 2015;91(4):234-7.

31. Dudareva-Vizule S, Haar K, Sailer A, Wisplinghoff H, Wisplinghoff F, Marcus U, et al. Prevalence of pharyngeal and rectal Chlamydia trachomatis and Neisseria gonorrhoeae infections among men who have sex with men in Germany. Sex Transm Infect. 2014;90(1):46-51.

32. Unemo M, Dillon JA. Review and international recommendation of methods for typing neisseria gonorrhoeae isolates and their implications for improved knowledge of gonococcal epidemiology, treatment, and biology. Clin Microbiol Rev. 2011;24(3):447-58.

33. Chisholm SA, Unemo M, Quaye N, Johansson E, Cole MJ, Ison CA, Van de Laar MJ. Molecular epidemiological typing within the European Gonococcal Antimicrobial Resistance Surveillance Programme reveals predominance of a multidrug-resistant clone. Euro Surveill. 2013;18(3). Available online: http://www.eurosurveillance.org/NiewArticle.aspx?Articleld=20358.

34. Jeverica S, Golparian D, Maticic M, Potocnik M, Mlakar B, Unemo M. Phenotypic and molecular characterization of Neisseria gonorrhoeae isolates from Slovenia, 2006-12: rise and fall of the multidrug-resistant NG-MAST genogroup 1407 clone? J Antimicrob Chemother. 2014;69(6):1517-25.

35. Morita-Ishihara T, Unemo M, Furubayashi K, Kawahata T, Shimuta K, Nakayama S, et al. Treatment failure with $2 \mathrm{~g}$ of azithromycin (extendedrelease formulation) in gonorrhoea in Japan caused by the international multidrug-resistant ST1407 strain of Neisseria gonorrhoeae. J Antimicrob Chemother. 2014;69(8):2086-90.

36. Galarza PG, Alcala B, Salcedo C, Canigia LF, Buscemi L, Pagano I, et al. Emergence of high level azithromycin-resistant Neisseria gonorrhoeae strain isolated in Argentina. Sex Transm Dis. 2009;36(12):787-8.

37. Palmer HM, Young H, Winter A, Dave J. Emergence and spread of azithromycin-resistant Neisseria gonorrhoeae in Scotland. J Antimicrob Chemother. 2008;62(3):490-4.

38. Chisholm SA, Dave J, Ison CA. High-level azithromycin resistance occurs in Neisseria gonorrhoeae as a result of a single point mutation in the $23 \mathrm{~S}$ rRNA genes. Antimicrob Agents Chemother. 2010;54(9):3812-6.

39. Starnino S, Stefanelli P. Neisseria gonorrhoeae Italian study G. Azithromycinresistant Neisseria gonorrhoeae strains recently isolated in Italy. J Antimicrob Chemother. 2009:63(6):1200-4

40. Katz AR, Komeya AY, Soge OO, Kiaha MI, Lee MV, Wasserman GM, et al. Neisseria gonorrhoeae with high-level resistance to azithromycin: case report of the first isolate identified in the United States. Clin Infect Dis. 2012;54(6):841-3.

41. Unemo M, Golparian D, Hellmark B. First three Neisseria gonorrhoeae isolates with high-level resistance to azithromycin in Sweden: a threat to currently available dual-antimicrobial regimens for treatment of gonorrhea? Antimicrob Agents Chemother. 2014;58(1):624-5.

42. Berçot B, Belkacem A, Goubard A, Mougari F, Sednaoui P, La Ruche G, Cambau E. High-level azithromycin-resistant Neisseria gonorrhoeae clinical isolate in France, March 2014. Euro Surveill. 2014;19(44). https://doi.org/10. 2807/1560-7917.ES2014.19.44.20951

43. Chisholm SA, Wilson J, Alexander S, Tripodo F, Al-Shahib A, Schaefer U, et al An outbreak of high-level azithromycin resistant Neisseria gonorrhoeae in England. Sex Transm Infect. 2016;92:365-367.

44. Tapsall J. Antimicrobial resistance in Neisseria gonorrhoeae, WHO collaborating Centre for STD and HIV. Sydney: World Health; 2001.

45. Buono SA, Watson TD, Borenstein LA, Klausner JD, Pandori MW, Godwin HA Stemming the tide of drug-resistant Neisseria gonorrhoeae: the need for an individualized approach to treatment. J Antimicrob Chemother. 2015;70(2): 374-81. 